\title{
86 EXTENSIVE FAP EXPRESSION ANALYSIS IN 23 TUMOR INDICATIONS AND POTENTIAL APPLICATION IN DEFINING THE PATIENT POPULATION IN FAP- TARGETING CANCER IMMUNOTHERAPIES
}

Sebastian Dziadek*, Anton Kraxner, Wei-Yi Cheng, Mike Flores, Tai-Hsien, Noah Theiss, Tsu-Shuen Tsao, Emilia Andersson, Suzana Vega Harring, Gabriele Gabriele Hoelzlwimmer, Ann-Marie Broeske, Maurizio Ceppi, Jehad Charo. Roche/Genentech, Basel, Switzerland

Background Fibroblast activation protein alpha (FAP) is frequently over-expressed in the tumor microenvironment (TME) while exhibiting limited expression in normal tissues. FAP expression was reported to be immunosuppressive in tumor mouse models and generally associated with worse prognosis in clinical studies. Therefore, it is important to understand the context in which FAP both exhibits immunosuppressive characteristics and be a useful target for immunotherapy.

Methods Comprehensive immunohistochemistry (IHC) analyses on formalin-fixed paraffin-embedded tissue specimens with emphasis on lymph nodes and primary and metastatic tumor lesions spanning a wide range of indications were undertaken in this study. FAP staining of tumor tissues was performed with an optimized IHC robust-prototype-assay (RPA) and manually scored. The area (normal stroma $\&$ neoplastic) staining positively relative to the total tumor area at each intensity level was recorded and an $\mathrm{H}$-score calculated (FAP-intensity score).These were supplemented by gene expression analysis using public as well as Roche phase 1, 2 and 3 cancer immunotherapy (CIT) clinical trial data sets.

Results Analysing FAP expression on normal tissue confirmed the general absence of FAP apart from a subset of pancreatic islet cells. Unlike the more homogenous expression of typical protein targets on tumor cells, FAP expression in the TME is heterogeneous in both pattern and intensity, requiring the analysis of a large sample set. Therefore, we evaluated 1216 samples from 23 tumor indications and 70 sub-indications. FAP expression exhibited a significant spread ranging from indications with highly abundant expression to those with low coverage.Using data from matching IHC and gene expression samples we confirmed FAP mRNA expression to significantly correlate with RPA H-scores (Spearman correlation: 0.62) $(\mathrm{N}=289, \mathrm{P}=1.2 \mathrm{E}-31)$. Gene expression data from 12 atezolizumab clinical studies, including standard of care (SOC) randomized studies, with more than 6000 samples from 4 major indications were interrogated for the association between FAP expression and clinical response as evaluated by overall and progression free survival. This analysis suggests that FAP expression is generally associated with higher hazard ratios across all atezolizumab-treated samples (OS: 95\% CI 1.04-1.09; PFS: 1.04-1.08), with the highest effect observed in Renal Cell Carcinoma (OS: 95\% CI 1.08-1.31; PFS: 1.051.21), indicating a potential role of FAP in limiting CIT.

Conclusions Data from these analyses can tailor indication and patient enrichment strategies for achieving optimal FAP-targeting. We propose to select indications with FAP-levels that are high enough to enable drug accumulation, yet low enough to reduce immunosuppressive effects that can hamper successful immunotherapy.

http://dx.doi.org/10.1136/jitc-2021-SITC2021.086 